“(C) 2018 IEEE. Personal use of this material is permitted. Permission from IEEE must be obtained for all other uses, in any current or future media, including reprinting/republishing this material for advertising or promotional purposes, creating new collective works, for resale or redistribution to servers or lists, or reuse of any copyrighted component of this work in other works." 


\section{Design of Dual Linearly Polarized, Electrically Small, Low-Profile, Broadside Radiating, Huygens Source Antenna}

\author{
Zhentian Wu, Ming-Chun Tang \\ College of Communication Engineering \\ Chongqing University \\ Chongqing, 400044, China \\ tangmingchun@cqu.edu.cn
}

\author{
Richard W. Ziolkowski \\ Global Big Data Technologies Centre \\ University of Technology Sydney \\ Ultimo NSW 2007, Australia \\ Richard.Ziolkowski@uts.adu.au
}

\begin{abstract}
A design of electrically small, low-profile, dual linearly polarized (LP), Huygens source antenna with high isolation, and broadside radiation patterns with high front forward radiation and low back forward radiation, i.e., high front-to-back ratios (FTBRs), is presented. A prototype dual-LP antenna was fabricated, assembled, and tested. The measured results, in good agreement with their simulated values, confirm that it has an electrically small size $(k a=0.904)$ and low profile $\left(0.0483 \lambda_{0}\right)$, with port isolation over $25.8 \mathrm{~dB}$ within its fractional impedance bandwidth (FBW), 0.46\%, where $\left|S_{11}\right|<-10 \mathrm{~dB}$. When port 1 (port 2) is excited, the peak realized gain is $2.03 \mathrm{dBi}(2.15$ $\mathrm{dBi}$ ) strictly along the broadside direction with the corresponding FTBR being $12.4 \mathrm{~dB}(12.1 \mathrm{~dB})$.
\end{abstract}

Keywords-Broadside radiation, cardioid radiation pattern, dual polarization, electrically small antennas, Huygens source antenna, near-field resonant parasitic elements

\section{INTRODUCTION}

With the development drive of wireless communication systems towards compactness and integration, electrically small antennas (ESAs) have attracted considerable attention in recent years. However, their directional radiation performance is generally constrained to be that of an electric or magnetic dipole antenna [1]. Consequently, their peak realized gains and front-to-back ratios (FTBRs) are usually quite low. In order to empower better directivity for a variety of wireless applications, e.g., long-distance/point-to-point wireless communications, there are several approaches that have been reported. These include loading the radiating elements with electromagnetic band gap (EBG) structures [2] and adding slot structures [3], only to name a few. Compared with the above reported approaches, the method of combining pairs of magnetic and electric dipoles to realize Huygens source ESAs holds inherent advantages of directivity enhancement without significantly increasing the overall size and profile of the antenna systems. Existing Huygens source ESAs can be classified into the two usual polarization categories: linearly polarized (LP) designs [4-6] and circularly polarized (CP) designs [7].

As is well known, the dual-polarized antennas process has a significant advantage over the single-polarized counterpart in many practical engineering applications. They have been extensively studied. A dual-polarized antenna design is equivalent to the combination of two different polarized antenna units. Consequently, it accordingly reduces the unit number and cost of the system while effectively increasing the communications capability and quality. For any typical dualpolarized antenna, high port isolation is an indispensable feature. Many effective structures/technologies have been tested to accomplish this feature, including, for example, aperture-coupled feed networks [8], meandering or crossprobes [9], coupled feed networks [10], and even-odd mode feed structures [11]. These structures/technologies inescapably make their total electric size not small.

In this paper, a dual-polarized, electrically small, lowprofile, Huygens source antenna with LP radiation performance characteristics is reported. The dual-LP design is introduced in Section II. A prototype of its optimized configuration was fabricated and assembled. The measured results are shown to be in good agreement with their simulated values. Finally, some conclusions are drawn in Section III.

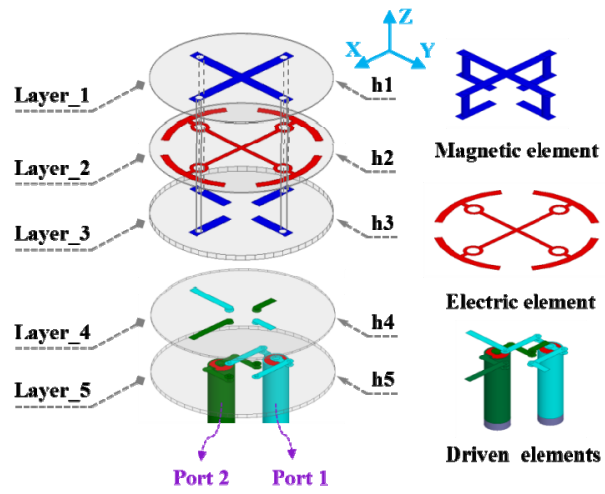

Fig.1. 3-D isometric view of the dual-LP Huygens source ESA.

\section{DUAL-LP HUYGENS SOURCE ESA}

The geometric structure of the dual-LP Huygens source ESA is shown in Fig. 1. It is a multi-layered structure, consisting of five substrate layers. All of the substrates are realized with the Rogers $4350 \mathrm{~B}$ board material with a copper 
thickness of $0.017 \mathrm{~mm}$, relative dielectric constant $\varepsilon_{r}=3.48$ and loss tangent $\tan \delta=0.0037$. All these substrate layers have the same radius $(28.5 \mathrm{~mm})$, but have different thicknesses: $\mathrm{h} 1=\mathrm{h} 2=\mathrm{h} 4=0.254 \mathrm{~mm}, \mathrm{~h} 3=1.508 \mathrm{~mm}, \mathrm{~h} 5=0.508 \mathrm{~mm}$.

The magnetic element consists of two orthogonally-oriented capacitively loaded loops (CLLs), one being oriented along the $x$-axis and the other along the $y$-axis. As a whole, the magnetic element is located on the upper surface of Layer_1 and Layer 3. The electric element is located on the upper surface of Layer_2, and consists of two Egyptian axe dipoles (EADs) along the $x$ - and $y$-axis, respectively. Both the dual CLLs and EADs operate as NFRP elements and are excited by driven dipole strips, which are located on Layer_4 and Layer_5. The operating mechanisms of the CLL and EAD NFRP elements, and the design guideline to realize a singlepolarized Huygens source ESA have been extensively described in [4]. In the present dual-LP design, the CLL and EAD elements located along the $y$-axis are both excited by port 1 to generate a Huygens source radiation pattern with linear polarization along the $y$-axis. Likewise, under the excitation of port 2, the combination of the CLL and EAD elements along the $x$-axis generate a second Huygens source radiation pattern with linear polarization along the $x$-axis.

\section{A. Simulated and Measured Dual-LP ESA Results}

The dual-LP ESA in Fig. 1 was fabricated, assembled and measured. The assembled dual-LP ESA prototype was mounted on two sleeve baluns, one for each port, as shown in Fig. 2.

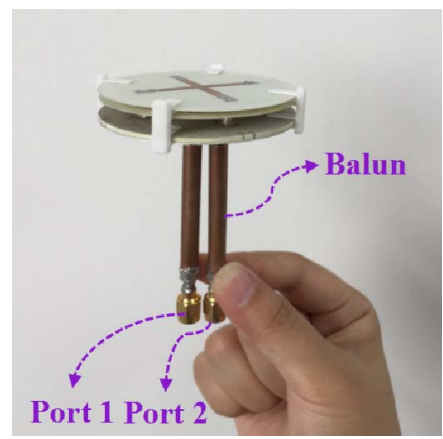

Fig. 2. Fabricated prototype of the dual-LP Huygens source ESA.

The simulated and measured results of the dual-LP Huygens source ESA are shown in Fig. 3. The simulated (measured) results demonstrate that the resonance frequencies of both states of the dual-LP ESA are centered at $1.518 \mathrm{GHz}$ $(1.515 \mathrm{GHz})$ with $\left|\mathrm{S}_{11}\right|_{\min }=-27.8 \mathrm{~dB}(-17.8 \mathrm{~dB})$ and $\left|\mathrm{S}_{22}\right|_{\min }=$ $-36.5 \mathrm{~dB}(-12.4 \mathrm{~dB})$. The corresponding impedance bandwidth was $9 \mathrm{MHz}(7 \mathrm{MHz})$. The isolation between the two ports is better than $30.6 \mathrm{~dB}(25.8 \mathrm{~dB})$ within the operational bandwidth. Thus, the simulated (measured) total height and electrical size of this dual-LP ESA at the resonance frequency were $0.0484 \lambda_{0}\left(0.0483 \lambda_{0}\right)$ and $\mathrm{ka}=0.906(0.904)$, respectively. These measured results are in good agreement with their simulated values; they confirm that the measured dual-LP Huygens source ESA is low-profile and electrically small.

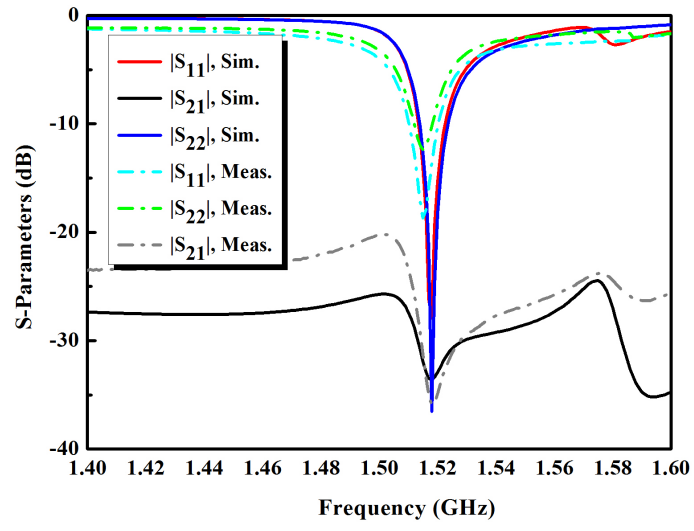

Fig. 3. Simulated and measured S-parameters of the dual-LP Huygens source ESA.

The simulated and measured radiation patterns of the dualLP ESA are shown in Fig. 4. It is clear that good broadside radiation performance was obtained. The main-beam directions for each polarization state are oriented along the $+\mathrm{z}-$ axis. Specifically, when port 1 was excited, the simulated (measured) peak realized gain value was $2.2 \mathrm{dBi}(2.03 \mathrm{dBi})$ and the FTBR value was $15.6 \mathrm{~dB}(12.4 \mathrm{~dB})$ at $1.518 \mathrm{GHz}$ $(1.515 \mathrm{GHz})$. The simulated radiation efficiency (RE) value corresponding to the peak realized gain value was $61.3 \%$. The simulated (measured) half-beam coverage was from $-60.8^{\circ}$ to $75.6^{\circ}\left(-68^{\circ}\right.$ to $\left.56^{\circ}\right)$ in $E$-plane ( $z 0 y$-plane) and from $-73.9^{\circ}$ to $75.9^{\circ}\left(-78^{\circ}\right.$ to $\left.80^{\circ}\right)$ in $H$-plane (zOx-plane). In contrast, when port 2 was excited, the simulated (measured) peak realized gain value was $2.5 \mathrm{dBi}(2.15 \mathrm{dBi})$ and the FTBR value was 16 $\mathrm{dB}(12.1 \mathrm{~dB})$ at $1.518 \mathrm{GHz}(1.515 \mathrm{GHz})$. The simulated radiation efficiency (RE) value corresponding to the peak realized gain value was $64 \%$. The simulated (measured) halfbeam coverage was from $-73.7^{\circ}$ to $66.6^{\circ}\left(-68^{\circ}\right.$ to $\left.46^{\circ}\right)$ in $E$ plane $\left(z 0 x\right.$-plane) and from $-70.8^{\circ}$ to $74.4^{\circ}\left(-134^{\circ}\right.$ to $\left.50^{\circ}\right)$ in $\mathrm{H}$ plane (z0y-plane).

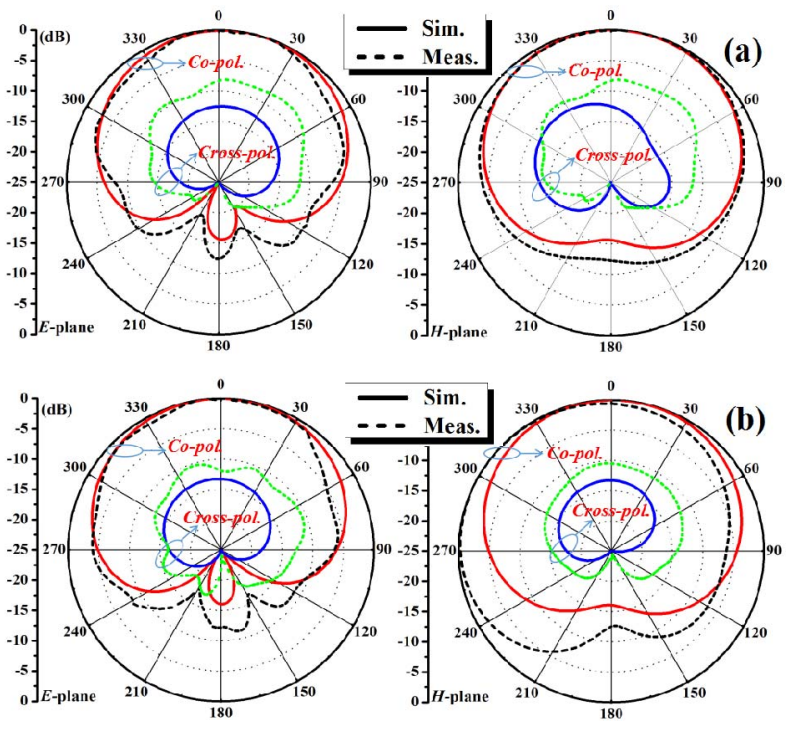

Fig. 4. Simulated and measured normalized realized gain patterns for each port in the $E$ - and $H$ - planes of the dual-LP Huygens source ESA. (a) Only port 1 is excited. (b) Only port 2 is excited. 


\section{CONCLUSION}

In this paper, a dual-LP Huygens source ESA was reported. It is low profile, has wide beamwidths, has high FTBR values, and exhibits high isolation between their two ports. It is anticipated that this dual-LP Huygens source ESA would be very good candidates for future wireless applications needing high performance, multi-functional, compact elements.

\section{References}

[1] R. F. Harrington, "Effect of antenna size on gain, bandwidth and efficiency," J. Res. Nat. Bur. Stand, vol. 64-D, pp. 1-12, Jan. / Feb. 1960.

[2] J. Ng, and R. W. Ziolkowski, "Combining metamaterial-inspired electrically small antennas with electromagnetic band gap (EBG) structures to achieve higher directivities and bandwidths," Proc. 2012 International Workshop on Antenna Technology (iWAT 2012), Tucson, America, pp. 189-192, Mar. 2012.

[3] M.-C. Tang, R. W. Ziolkowski, "Efficient, high directivity, large front-to-back-ratio, electrically small, near-field-resonant-parasitic antenna," IEEE Access, vol. 1, no. 1, pp. 16 - 28, May 2013.

[4] M.-C. Tang, H. Wang, and R. W. Ziolkowski, "Design and testing of simple, electrically small, low-profile, Huygens source antennas with broadside radiation performance," IEEE Trans. Antennas Propag., vol.64, no. 11, pp. 4607-4617, Nov. 2016.

[5] M.-C. Tang, T. Shi, and R. W. Ziolkowski, "Electrically small, broadside radiating Huygens source antenna augmented with internal non-Foster elements to increase its bandwidth," IEEE Antennas Wirel. Propag. Lett., vol.16, pp. 712-715, 2017.

[6] M.-C. Tang, B. Zhou, and R. W. Ziolkowski, "Low-profile, electrically small, Huygens source antenna with patternreconfigurability that covers the entire azimuthal plane," IEEE Trans. Antennas Propag., vol.65, no. 3, pp. 1063-1072, Mar. 2017.

[7] M.-C. Tang, T. Shi, R. W. Ziolkowski, "A study of $28 \mathrm{GHz}$, planar, multi-layered, electrically small, broadside radiating, Huygens source antennas," IEEE Trans. Antennas Propag., Special Issue on "Antennas and Propagation Aspects of 5G Communications" DOI: 10.1109/TAP.2017.2700888, Accepted on Apr. 02, 2017.

[8] M. Barba, "A high-isolation, wideband and dual-linear polarization," IEEE Trans. Antennas Propag., vol. 56, no. 5, May. 2008.

[9] H. W. Lai and K. M. Luk, "Dual polarized patch antenna fed by meandering probes," IEEE Trans. Antennas Propag., vol. 55, no. 9, pp. 2625-2627, Sep. 2007.

[10] H. Wong, K. L. Lau, and K. M. Luk, "Design of dual-polarized Lprobe patch antenna arrays with high isolation," IEEE Trans. Antennas Propag., vol. 52, no. 1, pp. 45-52, Jan. 2004.

[11] Y. Li, Z. Zhang, W. Chen, Z. Feng, and M. F. Iskander, "A dualpolarization slot antenna using a compact CPW feeding structure," IEEE Antennas Wirel. Propag. Lett., vol. 9, pp. 191-194, 2010. 